\title{
Clinical utility of F-18 sodium fluoride PET/CT for estimating disease activity in patients with rheumatoid arthritis
}

\author{
Hee Jin Park ${ }^{1 \wedge}$, Sung Hae Chang ${ }^{2 \wedge}$, Jeong Won Lee ${ }^{3} \wedge$, Sang Mi Lee ${ }^{4} \wedge$ \\ ${ }^{1}$ Division of Rheumatology, Department of Internal Medicine, Catholic Kwandong University College of Medicine, International St. Mary's \\ Hospital, 25, Simgok-ro 100-gil, Seo-gu, Incheon, Korea; ${ }^{2}$ Division of Rheumatology, Department of Internal Medicine, Soonchunhyang University \\ Cheonan Hospital, 31 Suncheonhyang 6-gil, Dongnam-gu, Cheonan, Chungcheongnam-do, Korea; ${ }^{3}$ Department of Nuclear Medicine, Catholic \\ Kwandong University College of Medicine, International St. Mary's Hospital, 25, Simgok-ro 100-gil, Seo-gu, Incheon, Korea; ${ }^{4}$ Department of \\ Nuclear Medicine, Soonchunhyang University Cheonan Hospital, 31 Suncheonhyang 6-gil, Dongnam-gu, Cheonan, Chungcheongnam-do, Korea
}

Correspondence to: Jeong Won Lee. International St. Mary's Hospital, 25, Simgok-ro 100-gil, Seo-gu, Incheon, 22711, Korea. Email: jwlee223@ish.ac.kr; Sang Mi Lee. Soonchunhyang University Cheonan Hospital, 31 Suncheonhyang 6-gil, Dongnam-gu, Cheonan, Chungcheongnam-do, 31151, Korea. Email: gareen@naver.com.

Background: The present study aimed to investigate the clinical implication of F-18 sodium fluoride $(\mathrm{NaF})$ positron emission tomography/computed tomography (PET/CT) for assessing the disease activity of rheumatoid arthritis.

Methods: Seventeen patients with rheumatoid arthritis according to the 2010 American College of Rheumatology/European League Against Rheumatism classification criteria were prospectively enrolled. All enrolled patients underwent F-18 NaF PET/CT along with physical examination, blood test, and ultrasonography. On PET/CT images, two quantitative parameters, F-18 NaF uptake of the joint (joint SUV) and joint-to-bone uptake ratio, were measured for each of the 28 joints included in calculating the disease activity score in 28 joints using erythrocyte sedimentation rate (DAS28-ESR). The relationship between PET/CT parameters and clinical factors and the predictive values of PET/CT parameters for joints with synovitis and high disease activity were evaluated.

Results: Tender joints (joint SUV, 13.6 \pm 8.4 ; joint-to-bone uptake ratio, $1.70 \pm 1.02$ ) and both tender and swollen joints (joint SUV, 13.9 \pm 5.4 ; joint-to-normal bone uptake ratio, $1.81 \pm 0.76$ ) had significantly higher joint SUV and joint-to-bone uptake ratio than joints without synovitis (joint SUV, 6.0 2.4; joint-tobone uptake ratio, $0.74 \pm 0.31 ; \mathrm{P}<0.001)$. On correlation analysis, summed joint $\mathrm{SUV}(\mathrm{P}=0.002$, correlation coefficient $=0.705)$ and summed joint-to-bone uptake ratio $(\mathrm{P}<0.001$, correlation coefficient=0.861) of 28 joints showed strong positive correlation with DAS28-ESR after adjustment for age and body mass index. Summed joint SUV showed significant positive correlations with ultrasonography findings (grey scale ultrasonography: $\mathrm{P}=0.047$, correlation coefficient $=0.468$; power Doppler ultrasonography: $\mathrm{P}=0.045$, correlation coefficient $=0.507$ ). On the receiver operating characteristic curve analysis, the sensitivity and specificity for predicting synovitis were $83.2 \%$ and $92.7 \%$, respectively, for joint SUV and $81.5 \%$ and $90.7 \%$, respectively, for joint-to-bone uptake ratio. Moreover, the summation of both PET/CT parameters of 28 joints showed a diagnostic accuracy of $100.0 \%$ for predicting high disease activity in rheumatoid arthritis.

Conclusions: Summed joint uptake on F-18 NaF PET/CT had a strong positive correlation with DAS28ESR and accurately predicted high disease activity. F-18 NaF PET/CT parameters might be used as an imaging biomarker for disease activity in rheumatoid arthritis.

Trial registration: This study was registered at the Clinical Research Information Service of the Korea (CRIS, http://cris.nih.go.kr/cris/en; registry number, KCT0002597; registered November 2017).

^ORCID: Hee Jin Park, 0000-0002-6324-5262; Sung Hae Chang, 0000-0002-7980-7194; Jeong Won Lee, 0000-0002-2697-3578; Sang Mi Lee, 0000-0002-7943-3807. 
Keywords: DAS28; F-18 sodium fluoride (NaF); positron emission tomography (PET); rheumatoid arthritis

Submitted Jun 23, 2020. Accepted for publication Oct 27, 2020.

doi: $10.21037 /$ qims-20-788

View this article at: http://dx.doi.org/10.21037/qims-20-788

\section{Introduction}

Rheumatoid arthritis is a chronic systemic inflammatory disease (1). It begins with the inflammatory response of the innate and adaptive immune systems involving multiple joints, resulting in persistent inflammatory synovitis which further progresses to cartilage destruction, bone erosion, and joint destruction $(1,2)$. Currently, the treat-to-target concept has been used to manage rheumatoid arthritis $(3,4)$. It aims to achieve early clinical remission or at least low disease activity and maintain responses through continuous monitoring of disease activity and prompt treatment adjustment, as early diagnosis and subsequent early intervention are known to be associated with better physical function, quality of life, and radiological outcomes (3-5). Therefore, accurate assessment of disease activity is crucial to make early treatment decisions (4). In clinical practice, disease activity score in 28 joints (DAS28), clinical disease activity index, and simplified disease activity index have been used to evaluate the disease activity in rheumatoid arthritis (6). However, those clinical measures mainly depend on patients' subjective joint symptoms $(4,7)$. In previous studies, imaging modalities such as ultrasonography, magnetic resonance imaging (MRI), and bone scintigraphy were found to be superior to physical examination in detecting joint inflammation and assessing disease activity, suggesting a valuable role of imaging modalities in rheumatoid arthritis (7-10). Thus, diagnostic imaging examinations have been recommended for improving the accuracy of diagnosis and disease activity assessment of rheumatoid arthritis (8).

F-18 sodium fluoride $(\mathrm{NaF})$ is a radiopharmaceutical for positron emission tomography/computed tomography (PET/CT) imaging and has an excellent profile for skeletal imaging (11). Although uptake mechanism of F-18 NaF in the bone is similar to the diphosphonate agents used in bone scintigraphy, F-18 NaF PET/CT is known to have better spatial resolution and sensitivity than conventional bone scintigraphy $(11,12)$. Furthermore, in contrast to bone scintigraphy, the uptake of F-18 NaF on PET/CT images can be quantitatively analyzed and used as an objective parameter for evaluating the degree of pathological bony changes (13-16). In previous studies, F-18 NaF PET/CT demonstrated the significant clinical values in various bone and joint diseases, including detecting bone metastasis, diagnosing surgical site infection after orthopedic surgery, and evaluating disease activity in psoriatic arthritis and sacroiliitis (17-20). However, in previously published literature, only a few studies have evaluated the performance of F-18 NaF PET/CT in patients with rheumatoid arthritis $(21,22)$, and the clinical implications of bone PET/CT findings on evaluating disease activity in rheumatoid arthritis are not fully understood.

In the present study, we performed F-18 NaF PET/CT in prospectively enrolled patients with rheumatoid arthritis and evaluated the clinical values of quantitative parameters of F-18 NaF PET/CT for assessing the disease activity.

\section{Methods}

\section{Study population}

This was a single-center prospective diagnostic study performed at Catholic Kwandong University International St. Mary's Hospital. Seventeen patients (13 women and 4 men) with rheumatoid arthritis were enrolled in our study between November 2017 and August 2019 according to the following criteria: patients (I) who were diagnosed with rheumatoid arthritis based on the 2010 American College of Rheumatology/European League Against Rheumatism classification criteria for rheumatoid arthritis, (II) who underwent clinical and imaging evaluation for initial diagnostic work-up or the work-up of a flare-up of joint pain, swelling, and/or stiffness suggestive of potential disease relapse, and (III) who were age $\geq 18$ years. Patients who had a previous history of malignant disease or metabolic bone disease were excluded. On clinical evaluation of the patients, physical examination, plain radiography of bilateral hands, and blood tests including serum erythrocyte sedimentation rate (ESR), rheumatoid factor, and anticitrullinated protein antibody (ACPA) were performed initially. Based on these results, the disease activities of rheumatoid arthritis were assessed by calculating the DAS28 using ESR (DAS28- 
ESR). Afterward, all enrolled patients underwent F-18 $\mathrm{NaF}$ PET/CT and ultrasonography before starting or changing medication for rheumatoid arthritis. On ultrasonography, a total of 10 joints, bilateral wrist, 2 nd and 3rd metacarpo-phalangeal (MCP), and $2 \mathrm{nd}$ and $3 \mathrm{rd}$ proximal interphalangeal (PIP) joints, were examined in every patient using grey scale ultrasonography (GSUS) and power Doppler ultrasonography (PDUS).

\section{F-18 NaF PET/CT scan}

All patients provided informed consent for F-18 NaF PET/CT before the procedure. There were no special preparations for patients before PET/CT scanning. F-18 $\mathrm{NaF}$ PET/CT was performed using a dedicated PET/CT scanner (Biograph mCT 20 scanner, Siemens Healthineers, Knoxville, TN, USA). A dose of $185 \mathrm{MBq}$ F-18 NaF was intravenously administered. After mean uptake period of $57 \pm 5$ minutes, whole-body PET/CT images were acquired from vertex to feet. First, a non-contrast-enhanced CT scan was initially performed for attenuation correction at $80 \mathrm{~mA}$ and $100 \mathrm{kV}_{\mathrm{p}}$ with a slice thickness of $5 \mathrm{~mm}$. Next, a PET scan was performed at two minutes per bed position using a three-dimensional acquisition mode. Afterward, PET/CT images of bilateral hands and wrist joints area were additionally obtained $100 \pm 11$ minutes after the F-18 $\mathrm{NaF}$ injection. PET/CT scan of bilateral hands and wrist joints was performed with the patient prone with arms over head supported by a soft cushion. For a CT scan, the same settings as the whole-body PET/CT scan were used, and for a PET scan, one-bed position images were acquired for three minutes using a three-dimensional mode. All PET images were reconstructed with an iterative reconstruction algorithm and attenuation correction.

\section{Image analysis}

All F-18 NaF PET/CT images were evaluated by two experienced nuclear medicine physicians blinded to the clinical information. For the assessment of the degree of uptake on bone and joint, the standardized uptake value (SUV), which was calculated as [decay corrected activity $(\mathrm{kBq})$ per tissue volume $(\mathrm{mL})] /[$ injected activity $(\mathrm{kBq})$ per body mass (g)], was used as a descriptive indicator (13). For each patient, two imaging parameters, SUV of a joint (joint SUV) and joint-to-bone uptake ratio, were measured for each of the 28 joints which were included in the assessment of DAS28-ESR. For measuring radiotracer uptake of joint, a spheroid-shaped volume-of-interest (VOI) was manually drawn for each of the 28 joints (bilateral shoulder, elbow, wrist, MCP, PIP, and knee joints) and the maximum SUV of the joint was calculated and defined as joint SUV (Figure 1). For calculating joint-to-bone uptake ratio, uptake of the thoracic and lumbar spines was selected as the reference bone uptake. First, vertebral bodies that showed severe osteoarthritic change, compression fracture, or post-operative change were excluded from the selection. Afterward, the three largest vertebral bodies were selected from those remaining spines for ease of drawing VOIs. A spheroid-shaped VOI (at least $1.5 \mathrm{~cm}$ in size) was manually drawn over the three vertebral bodies of the thoracic and lumbar spines and an isocontour using a cut-off SUV of $75 \%$ of the maximum SUV of VOI was automatically produced within each VOI of the vertebral body (Figure 1). The cut-off SUV of $75 \%$ of the maximum SUV have shown good reproducibility between subjects for measuring mean SUV of the spine (23). Mean SUV of the voxels within the isocontour was defined as SUV of the vertebral body and the mean value of SUV of the three vertebral bodies was calculated. Using the joint SUV and mean SUVs of the vertebral bodies, the joint-to-bone uptake ratio was calculated for all 28 joints.

For ultrasonography, an experienced rheumatologist semi-quantitatively graded GSUS and PDUS findings from score 0 to score 3 for each of the selected 10 joints as described in previous studies $(24,25)$.

\section{Statistical analysis}

All enrolled patients were categorized into three groups according to the DAS28-ESR; high disease activity group (DAS28-ESR >5.1), moderate disease activity group $(3.2<$ DAS28-ESR $\leq 5.1)$, and low disease activity group (DAS28-ESR $\leq 3.2$ ). According to the results of Levene's test, Kruskal-Wallis test with post-hoc comparisons using Dunn's test was performed to evaluate the difference of two F-18 NaF PET/CT parameters (joint SUV and jointto-bone uptake ratio) between tender joints, swollen joints, and joints without synovitis, and between patient groups with high, moderate, and low disease activities. To evaluate relationships between summed bone PET/CT parameters and clinical factors including patient global assessment, DAS28-ESR, and ultrasonography scores, Spearman rank correlation coefficients were calculated after performing a normality test. On the correlation analysis with patient global assessment and DAS28-ESR, summed 
A

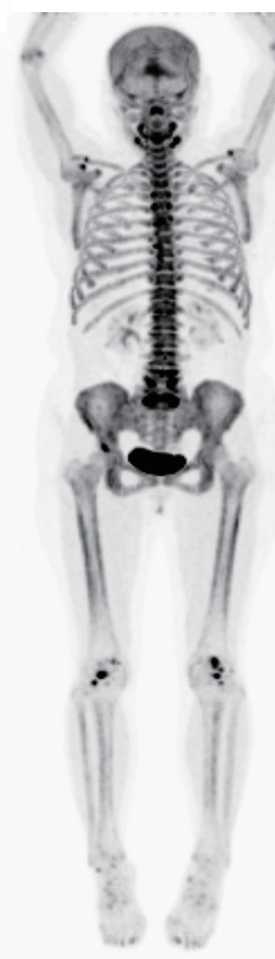

B

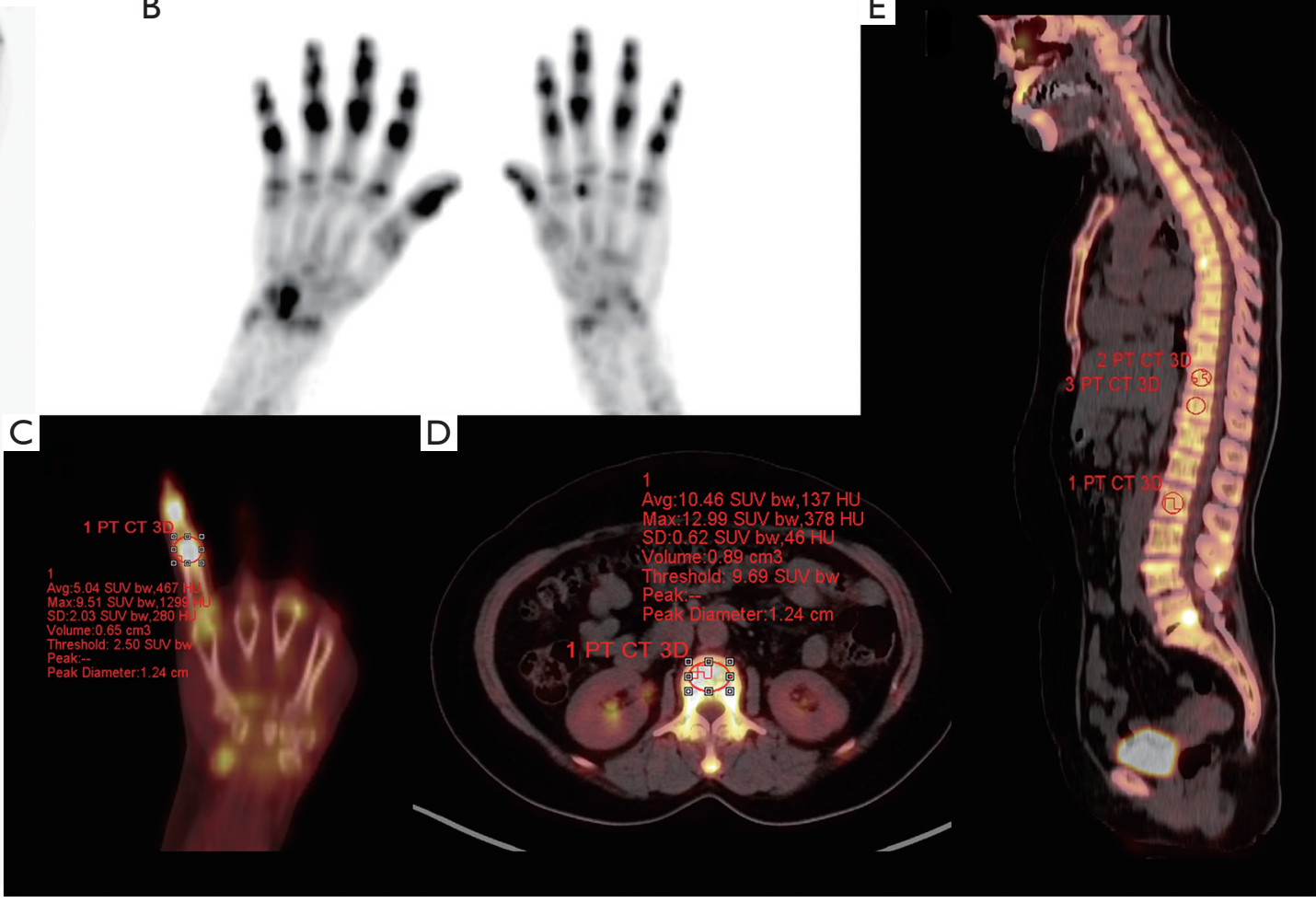

Figure 1 Maximum intensity projection images of whole-body (A) and hand and wrist area (B), fused coronal PET/CT image of the right hand (C), fused transaxial (D) and sagittal (E) PET/CT images of the body of a 65-year-old woman who underwent F-18 NaF bone PET/CT for the initial work-up of rheumatoid arthritis with DAS28-ESR of 6.28. On maximum intensity projection images, (A) and (B), multifocal increased periarticular uptake is observed in the bilateral elbow joints, bilateral wrist joints, left 3rd MCP joint, interphalangeal joints of bilateral hands, and bilateral knee joints. For measuring joint SUV, a spheroid-shaped VOI was manually drawn on the joint area, showing a measurement example of right 5th PIP joint on coronal PET/CT images (C) with a joint SUV of 9.51. For measuring reference bone uptake, three VOIs were manually drawn over the vertebral bodies of thoracic and lumbar spines, (D) and (E). An isocontour using a cut-off SUV value of $75 \%$ of the maximum SUV of VOI was automatically produced within each VOI of the vertebral body and mean SUV of voxels within the isocontour was measured. Using joint SUV and mean SUV of the vertebral bodies of the spine, joint-to-bone uptake ratio was calculated for each joint.

bone PET/CT parameters of 28 joints were used, while, on the correlation analysis with ultrasonography scores, summed PET/CT parameters of 10 joints (bilateral wrist, 2nd and 3rd MCP, and 2nd and 3rd PIP joints which were examined on ultrasonography) were used. All correlation analyses were performed after adjustment for age and body mass index. The predictive values of F-18 NaF PET/CT parameters for synovitis and high disease activity were investigated based on the area under the receiver operating characteristic (ROC) curve (AUC) values. Using the optimal cut-off values determined by the ROC curve analysis, the sensitivity and specificity of joint SUV and joint-to-bone uptake ratio for predicting joint with synovitis and those of summed joint SUV and joint-to-bone uptake ratio of 28 joints for predicting high disease activity were evaluated. All statistical analyses were performed using MedCalc Statistical Software version 19.5.3 (MedCalc Software Ltd, Ostend, Belgium). A P value of $<0.05$ was considered statistically significant.

\section{Results}

\section{Patient baseline characteristics}

The baseline characteristics of the 17 enrolled patients are shown in Table 1. One patient (5.9\%) underwent F-18 $\mathrm{NaF}$ PET/CT for initial work-up and the remaining 16 patients $(94.1 \%)$ were being treated for rheumatoid arthritis 
Table 1 Patient characteristics $(\mathrm{n}=17)$

\begin{tabular}{|c|c|c|}
\hline Characteristics & Number of patients (\%) & Median [range] \\
\hline Female & $13(76.5 \%)$ & \\
\hline \multicolumn{3}{|l|}{ Disease activity } \\
\hline Tender joint count (28 joints) & & $3[0-21]^{*}$ \\
\hline DAS28ESR & & $3.73[2.62-6.73]$ \\
\hline $\mathrm{ESR}(\mathrm{mm} / \mathrm{h})$ & & $21[7-120]$ \\
\hline \multicolumn{3}{|l|}{ Medications } \\
\hline Methotrexate & $8(47.1 \%)$ & \\
\hline Treatment duration (years) & & $3.8[0.5-6.0]$ \\
\hline \multicolumn{3}{|l|}{ Imaging } \\
\hline \multicolumn{3}{|l|}{ Ultrasonography } \\
\hline GSUS & & $8[2-13]^{\dagger}$ \\
\hline PDUS & & $2[0-5]^{\dagger}$ \\
\hline \multicolumn{3}{|l|}{ F-18 NaF bone PET-CT } \\
\hline Joint SUV & & $6.44[1.70-37.13]$ \\
\hline Summed joint SUV of 28 joints & & $190.86[98.02-331.71]$ \\
\hline
\end{tabular}

*, the number of tender or swollen joints among the 28 joints for each of the patients; ${ }^{\dagger}$, the sum of ultrasonography scores of the selected 10 joints for each patient. DAS28-ESR, disease activity score in 28 joints using erythrocyte sedimentation rate; ESR, erythrocyte sedimentation rate; GSUS, grey scale ultrasonography; PDUS, power Doppler ultrasonography; F-18 NaF, F-18 sodium fluoride; SUV, standardized uptake value.

at the time of PET/CT scanning. The median duration of treatment for rheumatoid arthritis in these 16 patients was 3.8 years (range, $0.5-6.0$ years). Of the 17 patients, 15 patients (88.2\%) were positive for rheumatoid factor, and 16 patients (94.1\%) were positive for ACPA. On the assessment with DAS28-ESR, 5 (29.4\%) patients were classified as high disease activity group (DAS28-ESR $>5.1), 8$ (47.1\%) were moderate disease activity group $(3.2<$ DAS28-ESR $\leq 5.1)$, and the remaining $4(23.5 \%)$ were low disease activity group (DAS28-ESR $\leq 3.2$ ). On physical examination, among 476 joints analyzed in the study, there were 34 tender joints, 3 swollen joints, and 41 joints that were both tender and swollen.

\section{Comparison of joint uptake}

Using joint SUVs and joint-to-bone uptake ratios of 28 joints, joint uptake on F-18 NaF PET/CT between tender joints (34 joints), both tender and swollen joints (41 joints), and joints without synovitis (398 joints) were compared. Because there were only three swollen joints, they were excluded from the analysis. On Kruskal-Wallis test, there were significant differences in both joint SUV and joint-to-bone uptake ratio among three joint groups $(\mathrm{P}<0.001$ for all; Figure 2). In post-hoc comparisons with Dunn's test, tender joints (joint SUV, 13.6 \pm 8.4 ; joint-tobone uptake ratio, $1.70 \pm 1.02)$ and both tender and swollen

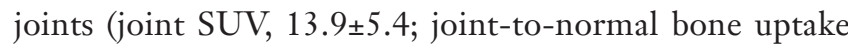



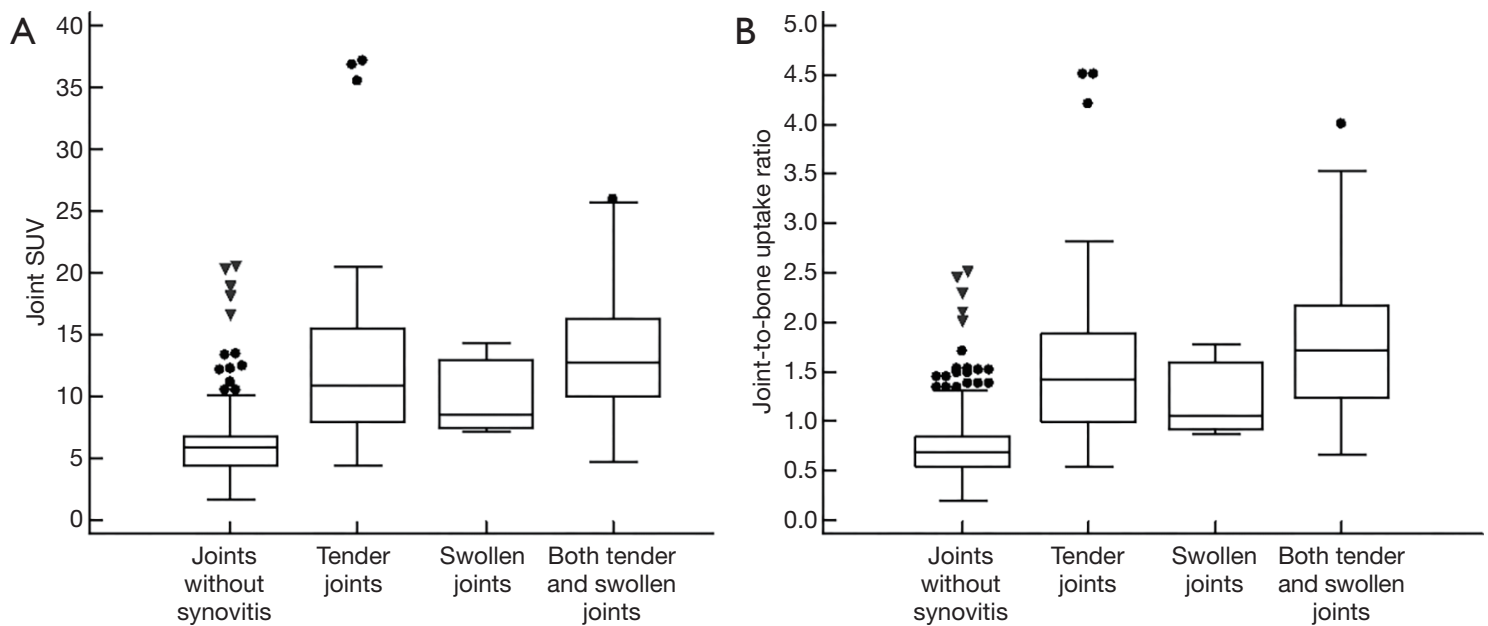

Figure 2 Distribution of the joint SUV (A) and joint-to-bone uptake ratio (B) in tender joints (34 joints), swollen joints (3 joints), both tender and swollen joints (41 joints), and joints without synovitis (398 joints).

Table 2 Comparison of F-18 NaF bone PET/CT parameters according to the disease activity of rheumatoid arthritis

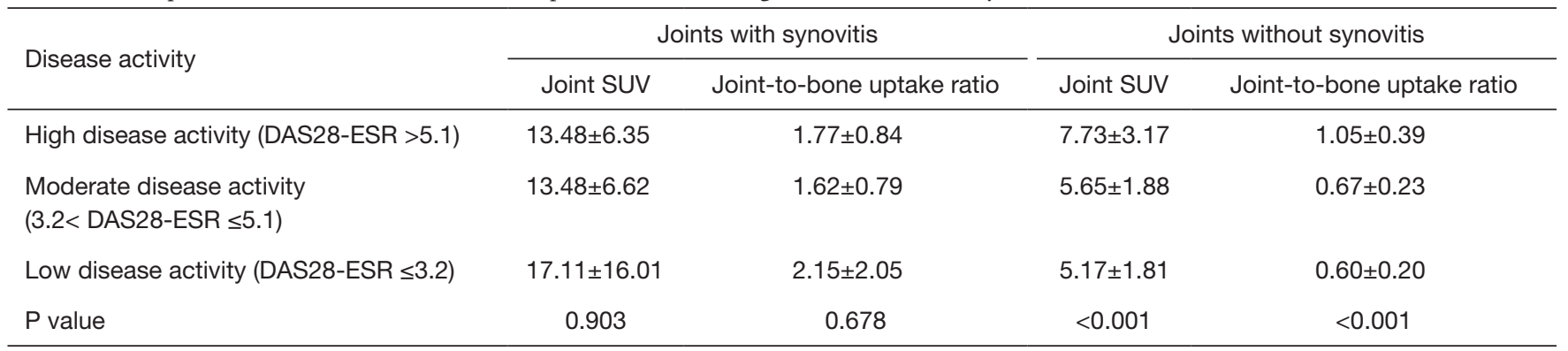

F-18 NaF, F-18 sodium fluoride; DAS28-ESR, disease activity score in 28 joints using erythrocyte sedimentation rate; SUV, standardized uptake value.

ratio, $1.81 \pm 0.76)$ showed significantly higher values of both joint SUV and joint-to-bone uptake ratio than joints without synovitis (joint SUV, 6.0 \pm 2.4 ; joint-to-bone uptake ratio, $0.74 \pm 0.31 ; \mathrm{P}<0.05$ for all). In contrast, there were no significant differences in both joint SUV and joint-to-bone uptake ratio between tender joints and both tender and swollen joints ( $\mathrm{P}>0.05$ for all).

We further compared joint SUV and joint-to-normal uptake ratios of joints with synovitis and joints without synovitis among patients with high, moderate, and low disease activities (Table 2). On Kruskal-Wallis test, no significant differences of joint SUV and joint-to-bone uptake ratio of joints with synovitis were shown between the three patient groups $(\mathrm{P}>0.05$; Figure $\mathrm{S} 1)$. However, there were significant differences in both joint SUV and joint-to- bone uptake ratio of joints without synovitis between groups $(\mathrm{P}<0.001$ for all; Figure $\mathrm{S} 1)$. In post-hoc comparisons, the patient group with high disease activity showed significantly higher joint SUV and joint-to-bone uptake ratio of the joints without synovitis than other groups $(\mathrm{P}<0.05$ for all). Meanwhile, there were no significant differences in joint SUV and joint-to-bone uptake ratio between patient groups with moderate and low disease activity $(\mathrm{P}>0.05)$.

\section{Correlation between summed joint uptake and clinical factors}

On correlation analysis with summed joint uptake on F-18 NaF PET/CT to clinical factors after adjustment for age and body mass index (Table 3), DAS28-ESR showed 
Table 3 Correlations of summed joint uptake on F-18 NaF bone PET/CT with patient global assessment, DAS28-ESR, GSUS, and PDUS after adjustment for age and body mass index.

\begin{tabular}{|c|c|c|}
\hline Clinical factors & Summed joint SUV & Summed joint-to-bone uptake ratio \\
\hline Correlation coefficient & $0.637^{*}$ & $0.646^{*}$ \\
\hline$P$ value & 0.008 & 0.007 \\
\hline \multicolumn{3}{|l|}{ DAS28-ESR } \\
\hline$P$ value & 0.002 & $<0.001$ \\
\hline \multicolumn{3}{|l|}{ GSUS } \\
\hline Correlation coefficient & $0.468^{\dagger}$ & $0.043^{\dagger}$ \\
\hline$P$ value & 0.047 & 0.066 \\
\hline$P$ value & 0.045 & 0.064 \\
\hline
\end{tabular}

${ }^{*}$, correlation analyses were performed with summed joint SUV or joint-to-bone uptake ratio of 28 joints; ${ }^{\dagger}$, correlation analyses were performed with summed joint SUV or joint-to-bone uptake ratio of bilateral wrist, 2nd and 3rd MCP, and 2nd and 3rd PIP joints that were examined in the ultrasonography. F-18 NaF, F-18 sodium fluoride; DAS28-ESR, disease activity score in 28 joints using erythrocyte sedimentation rate; GSUS, grey scale ultrasonography; PDUS, power Doppler ultrasonography; SUV, standardized uptake value.
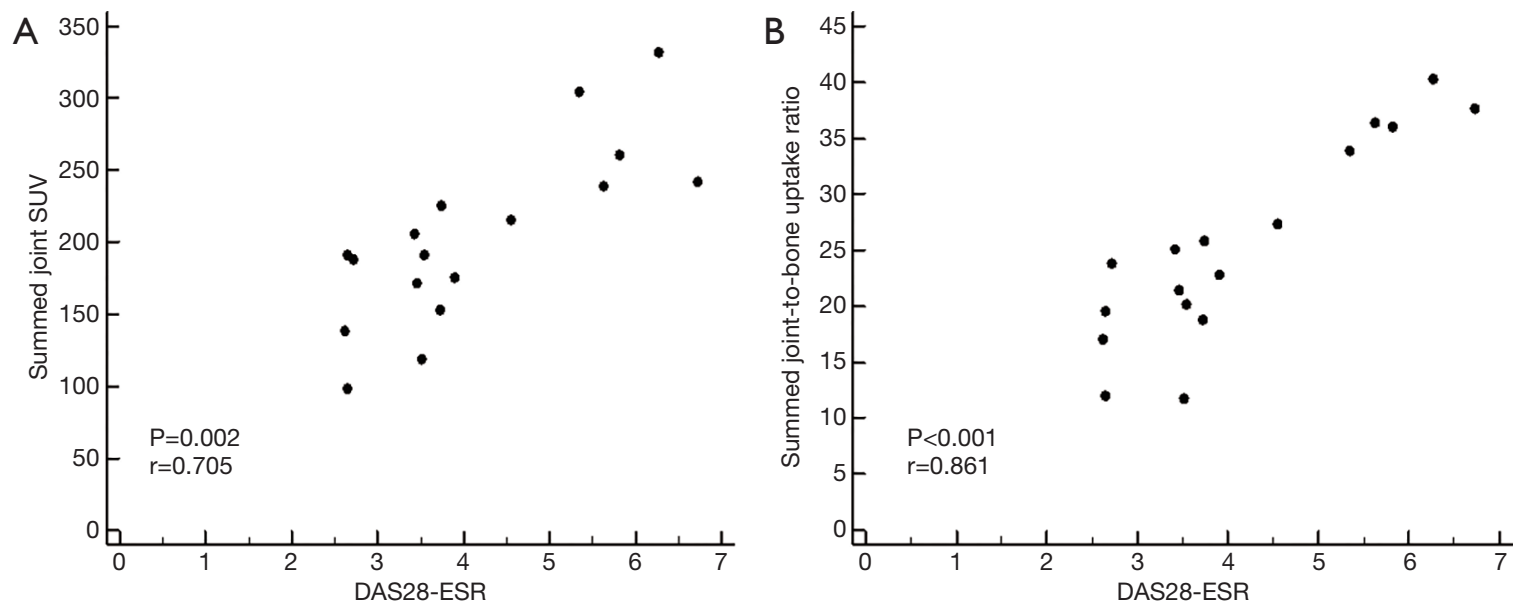

Figure 3 Scatter plots showing the relationship between DAS28-ESR and parameters of F-18 NaF bone PET/CT, summed joint SUV of 28 joints (A) and summed joint-to-bone uptake ratio of 28 joints (B).

strong positive correlations with both summed joint SUV $(\mathrm{P}=0.002$, correlation coefficient $=0.705)$ and joint-tobone uptake ratio $(\mathrm{P}<0.001$, correlation coefficient $=0.861)$ of the 28 joints (Figure 3). Furthermore, patient global assessment also showed significant moderate correlations with both summed joint SUV and joint-to-bone uptake ratio $(\mathrm{P}<0.05$ for all) of 28 joints. For ultrasonography findings, both GSUS and PDUS scores demonstrated significant positive correlations with summed joint SUV of the 10 joints that were included in the ultrasonography 

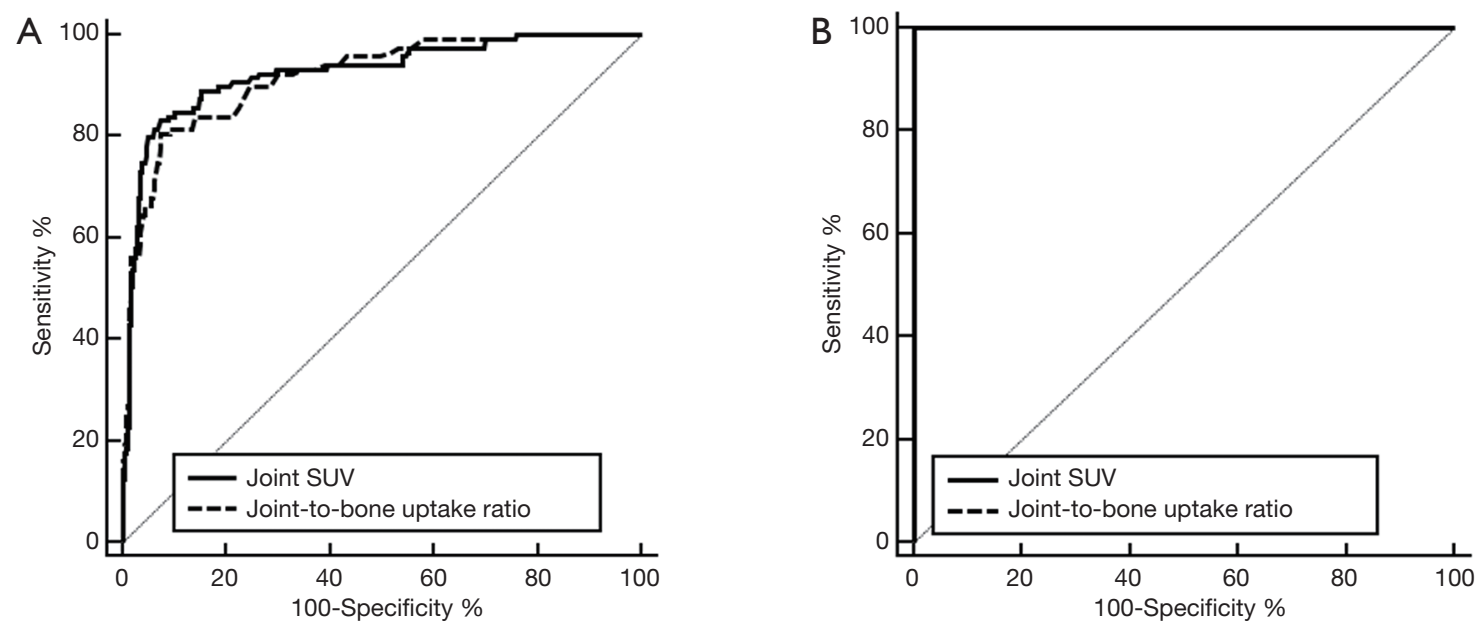

Figure 4 Receiver operating characteristic curves for joint SUV and joint-to-bone uptake ratio for predicting joint with synovitis (A) and for predicting high disease activity of rheumatoid arthritis (B).

examination ( $\mathrm{P}=0.047$ for GSUS and $\mathrm{P}=0.045$ for $\mathrm{PDUS}$ ), but only showed borderline significant correlations with joint-to-bone uptake ratio of the 10 joints $(\mathrm{P}=0.066$ for GSUS and $\mathrm{P}=0.064$ for PDUS).

\section{Predictive value for synovitis and high disease activity}

On ROC curve analysis, both joint SUV (AUC, 0.926; 95\% confidence interval, 0.900-0.947) and joint-to-bone uptake ratio (AUC, 0.919; 95\% confidence interval, 0.893-0.941) showed high values of AUC for predicting joints with synovitis (Figure 4A). Using the optimal cut-off values of joint SUV of 8.86 , the sensitivity and specificity of joint SUV for predicting synovitis were $83.2 \%$ (95\% confidence interval, $75.2-89.4 \%$ ) and $92.7 \%$ (95\% confidence interval, 89.7-95.1\%), respectively. For joint-to-bone uptake ratio, the sensitivity and specificity were $81.5 \%$ (95\% confidence interval, $73.4-88.0 \%$ ) and $90.7 \%$ (95\% confidence interval, 87.4-93.4\%), respectively, using the cut-off value of 1.05 .

On ROC curve analysis for predicting high disease activity in rheumatoid arthritis, both summed joint SUV and joint-to-bone uptake ratio of 28 joints showed an AUC value of 1.000 (95\% confidence interval, $0.805-1.000$ for both; Figure 4B). The optimal cut-off values determined by ROC curve analysis were 225.0 for summed joint SUV of the 28 joints and 28.0 for summed joint-to-bone uptake ratio of the 28 joints. Using these cut-off values, the sensitivity and specificity of both bone PET/CT parameters for predicting high disease activity in rheumatoid arthritis were $100.0 \%$ (95\% confidence interval, $47.8-100.0 \%$ for both) and $100.0 \%$ (95\% confidence interval, $73.5-100.0 \%$ for both), respectively.

\section{Discussion}

Uptake of F-18 NaF in a joint with synovitis in rheumatoid arthritis is known to be related to two pathological processes of the disease, hypervascularity and bone destruction (21). Ongoing bone erosion in rheumatoid arthritis leads to an increase of exposed bone surface, which results in increased incorporation of F-18 fluoride in the bone $(21,26)$. In a previous study with animal arthritis model, increased F-18 NaF accumulation was observed in the joint during the course of experimental arthritis, this was in contrast to no distinct F-18 NaF uptake of joint before the onset of arthritis, and the degree of F-18 NaF uptake was significantly related to the degree of bone destruction (26). In a previous clinical study on patients with rheumatoid arthritis, F-18 NaF accumulated in the erosive bone lesion of the joint with synovitis and increased F-18 NaF uptake was associated with progressive joint damage (21). Another previous study with rheumatoid arthritis patients also demonstrated that patients with increased F-18 NaF uptake in the knee joints had greater joint deterioration, showing a significant positive correlation between PET/ CT parameters and Kellgren-Lawrence grades (22). In our study, we directly compared joint uptake on F-18 NaF PET/CT according to the findings of physical examination 
and found that joints with synovitis had significantly higher F-18 NaF uptake than those without synovitis. Furthermore, the results of our study also revealed a significant positive correlation of joint SUV with GSUS and PDUS scores, which have already been known to be related to the degree of joint inflammation in rheumatoid arthritis $(27,28)$. Therefore, although F-18 NaF uptake cannot directly reflect the degree of inflammatory reaction (21), joint uptake on F-18 NaF PET/CT might be used to discriminate damaged joints by inflammation in patients with rheumatoid arthritis. Accordingly, both quantitative parameters of bone PET/CT showed high sensitivity and specificity for predicting joint with synovitis in the present study.

DAS2 8 has been considered as the gold standard for estimating disease activity and evaluating treatment response in rheumatoid arthritis at an individual level (29). However, the precise and objective assessment of all 28 joints is not easy to perform in routine clinical practice; thereby, imaging modalities have been often used for detecting joint inflammation and helping in making clinical decisions $(4,7,27)$. In the present study, summed joint SUV and joint-to-bone uptake ratio of 28 joints on F-18 NaF $\mathrm{PET} / \mathrm{CT}$ was found to have a significant strong positive correlation with DAS28-ESR, which was also reported in the previous study with bone PET/CT (21). In addition, usage of optimal cut-off values, summed 28 joints uptake successfully discriminated high disease activity patients with an accuracy of $100 \%$. Since patients with high disease activity would have large numbers of joints with synovitis, it is reasonable that those patients have greater summed joint uptake than others. On the other hand, the results of our study revealed that, contrary to the joints with synovitis, patients with high disease activity had significantly higher F-18 NaF uptake of joints without synovitis than other patients, which could also contribute to high summed joint uptake. Considering that F-18 NaF uptake is related to joint damage by inflammation $(21,26)$, this finding in our study might be an imaging evidence that, in rheumatoid arthritis patients with high disease activity, a large number of joints which seemed to be nonaffected or sustaining remission on physical examination could have subclinical inflammation and bone erosion. Furthermore, some of the joints without synovitis in patients with low and moderate disease activity demonstrated increased joint uptake, similar to those in patients with high disease activity, suggesting the presence of subclinical joint damage even in patients with low and moderate disease activity. Recently, there has been growing evidence that a significant portion of patients on remission or with low disease activity have subclinical synovitis (30,31). Because subclinical inflammation on imaging examinations was found to be associated with clinical outcomes, an attempt has been made to incorporate subclinical inflammation into treat-to-target strategies, although recent clinical trials have shown negative results $(30,32,33)$. Therefore, in addition to the role of aiding clinical assessment by differentiating patients with high disease activity, F-18 NaF PET/CT might be used as an imaging modality for detecting subclinical synovitis. Further longitudinal studies are needed to evaluate the clinical role of F-18 NaF uptake in assessing subclinical joint damage and managing subclinical inflammation of rheumatoid arthritis.

Plain radiography is the mainstay of imaging examination for rheumatoid arthritis, but cannot directly assess the degree of joint inflammation and has low sensitivity for small and subtle bone erosions $(4,27,34)$. Therefore, ultrasonography, MRI, and bone scintigraphy have been currently used as adjunct imaging modalities in rheumatoid arthritis, but each of these modalities has some intrinsic shortcomings $(7,8,35)$. Ultrasonography and MRI have high diagnostic utility in joint inflammation and for monitoring disease activity and treatment response (9,27,35-37). In contrast, evaluating all of the 28 joints with these imaging modalities is not applicable in routine clinical practice and there is no consensus regarding the minimum number of joints to be examined in rheumatoid arthritis $(7,27,35,37)$. Bone scintigraphy also has high sensitivity in detecting joint involvement and is able to assess all the joints of the body; however, the findings on bone scintigraphy had a limited relationship with disease activity and were found to have a limited benefit over clinical examination in rheumatoid arthritis (7,8,38-40). Most of all, the interpretation of the aforementioned imaging modalities is mainly dependent on visual analysis and quantitative analyses of findings are not currently available $(27,35,38)$. Thus, inter- and intra-reader discrepancies in visual image analysis are still a matter of concern $(25,27,35,41)$.

In this regard, the main strength of F-18 NaF PET/ CT imaging shown in this preliminary study is that the degree of joint involvement can be directly quantified, along with the ability to image whole-body joints and detect ongoing bone destruction (21). Using objective quantitative parameters measured on bone PET/CT, we could predict 
joints with synovitis and patients with high disease activity with high sensitivity and specificity, which might aid in the diagnosis of joint inflammation and assessment of disease status of rheumatoid arthritis. The results of our study suggest that F-18 NaF PET/CT might be used as another imaging method option for the assessment of patients with rheumatoid arthritis. Nevertheless, for considering the clinical use of bone PET/CT in rheumatoid arthritis, F-18 NaF PET/CT also has several inherent drawbacks. Still, the standard quantitative parameter for estimating the degree of abnormalities on PET/CT is not established $(13,18)$. Consequently, we measured two quantitative parameters in this study, SUV of joint itself and joint-tobone uptake ratio, which were comparable to each other in evaluating disease activity in rheumatoid arthritis. In addition to the parameters used in our study, several recent studies demonstrated the clinical usefulness of volumetric parameters, such as metabolically active volume, for assessing joint radiotracer uptake (42-44). Moreover, in joints without synovitis, there is a possibility of difficulty in distinguishing joint uptake from that of the surrounding bone, which might limit the accuracy of joint uptake measurement. Therefore, further discussion is required to determine the standard method of measuring quantitative parameters in bone PET/CT to best reflect the degree of joint damage. The number of joints to be included for bone PET/CT imaging analysis should be also determined. Furthermore, F-18 NaF also accumulates in pathologic joint conditions other than rheumatoid arthritis, such as osteoarthritis, which would limit the specificity of F-18 NaF PET/CT (21). However, ultrasonography, MRI, and bone scintigraphy also have similar diagnostic limitations resulting from a lack of specificity $(27,35,38,40)$. Another concern is that, because the uptake of F-18 NaF lasts even after arthritis subsides, it might be difficult to determine histopathologic status and treatment response solely based on F-18 NaF PET/CT results $(21,26)$. Therefore, further studies are needed to establish the clinical role of $\mathrm{F}-18$ $\mathrm{NaF}$ PET/CT in rheumatoid arthritis as compared to conventional imaging modalities.

Recently, several studies tried to investigate the clinical use of F-18 fluorodeoxyglucose (FDG) PET/CT in patients with rheumatoid arthritis $(21,43,45,46)$. Because of the upregulation of glycolytic activity of immune cells in inflammation, increased F-18 FDG uptake is observed in regional inflammation $(43,45)$. In rheumatoid arthritis, F-18 FDG uptake of the joint was reflective of glucose utilization of macrophages in the joint and showed a positive correlation with the degree of synovitis $(43,47)$. In several clinical studies, F-18 FDG PET/CT proved to be effective in differentiating rheumatoid arthritis from other arthritic diseases and assessing the disease activity in rheumatoid arthritis $(43,45,46)$. In another previous study that performed both F-18 NaF PET/CT and F-18 FDG $\mathrm{PET} / \mathrm{CT}$ in patients with rheumatoid arthritis, F-18 NaF uptake was mainly observed in erosive lesions of the bone cortex, whereas F-18 FDG uptake mainly accumulated in the surrounding soft tissue (21). Furthermore, the uptake pattern of both radiotracers did not exactly overlap, suggesting that both radiotracers are related to different aspects of rheumatoid arthritis (21). In future studies, the clinical significance of both radiotracers should be compared to establish the role of each radiotracer in rheumatoid arthritis.

This study has several unaddressed limitations. First, the present study included only a small number of patients selected from a single medical center; thus, our results should be further validated. Second, patients in our study were in heterogeneous clinical conditions on diverse medication, which might affect the results of F-18 NaF PET/CT images. Particularly, most of the patients $(94.1 \%)$ in our study were already receiving treatment for rheumatoid arthritis at the time of PET/CT scanning; thus, a further study would be needed with enrollment of patients upon initial work-up. Another limitation is that only the relationship of F-18 NaF PET/CT with clinical examination and disease activity was evaluated in this preliminary study. Further analysis that assesses clinical benefit over clinical examination and other imaging modalities should be performed in our future studies. Lastly, a positron emission mammography (PEM) was shown to have superior resolution and sensitivity for evaluating small bones and joints (48); hence, PEM could be more suitable for quantifying uptake of small joints in rheumatoid arthritis.

\section{Conclusions}

In this preliminary study, joints with synovitis showed significantly higher values of joint uptake on F-18 NaF PET/CT images than other joints, and summed joint uptake on bone PET/CT revealed a strong positive correlation with DAS28-ESR as well as a moderate positive correlation with ultrasonography findings in patients with rheumatoid arthritis. Furthermore, both joint SUV and joint-to-bone uptake ratio on PET/CT demonstrated high 
sensitivity and specificity for predicting joint with synovitis and high disease activity in rheumatoid arthritis. Our results thus suggest that joint uptake on F-18 NaF PET/CT might be used as an imaging biomarker for estimating disease activity in rheumatoid arthritis. However, further validation is warranted in future studies.

\section{Acknowledgments}

We would like to thank Editage (www.editage.co.kr) for English language editing.

Funding: This work was supported by Soonchunhyang University Research Fund and the National Research Foundation of Korea (NRF) grant funded by the Korea government (Ministry of Science and ICT) (grant number: NRF-2017R1C1B5075905).

\section{Footnote}

Conflicts of Interest: All authors have completed the ICMJE uniform disclosure form (available at http://dx.doi. org/10.21037/qims-20-788). The authors have no conflicts of interest to declare.

Ethical Statement: The trial was conducted in accordance with the Declaration of Helsinki. This prospective study was approved by the Institutional Review Board of Catholic Kwandong University International St. Mary's Hospital (IRB number: IS17ONSI0061). Informed consent for F-18 $\mathrm{NaF}$ bone PET/CT was obtained from all the patients enrolled in the present study before PET/CT scan. This study was registered at the Clinical Research Information Service of the Korea (CRIS, http://cris.nih.go.kr; registry number, KCT0002597).

Open Access Statement: This is an Open Access article distributed in accordance with the Creative Commons Attribution-NonCommercial-NoDerivs 4.0 International License (CC BY-NC-ND 4.0), which permits the noncommercial replication and distribution of the article with the strict proviso that no changes or edits are made and the original work is properly cited (including links to both the formal publication through the relevant DOI and the license). See: https://creativecommons.org/licenses/by-nc-nd/4.0/.

\section{References}

1. Alam J, Jantan I, Bukhari SNA. Rheumatoid arthritis: Recent advances on its etiology, role of cytokines and pharmacotherapy. Biomed Pharmacother 2017;92:615-33.

2. Grassi W, De Angelis R, Lamanna G, Cervini C. The clinical features of rheumatoid arthritis. Eur J Radiol 1998;27 Suppl 1:S18-24.

3. Smolen JS, Breedveld FC, Burmester GR, Bykerk V, Dougados M, Emery P, Kvien TK, Navarro-Compan MV, Oliver S, Schoels M, Scholte-Voshaar M, Stamm T, Stoffer M, Takeuchi T, Aletaha D, Andreu JL, Aringer $\mathrm{M}$, Bergman M, Betteridge N, Bijlsma H, Burkhardt $\mathrm{H}$, Cardiel M, Combe B, Durez P, Fonseca JE, Gibofsky A, Gomez-Reino JJ, Graninger W, Hannonen P, Haraoui B, Kouloumas M, Landewe R, Martin-Mola E, Nash P, Ostergaard M, Ostor A, Richards P, Sokka-Isler T, Thorne C, Tzioufas AG, van Vollenhoven R, de Wit M, van der Heijde D. Treating rheumatoid arthritis to target: 2014 update of the recommendations of an international task force. Ann Rheum Dis 2016;75:3-15.

4. Salomon-Escoto K, Kay J. The "Treat to Target" Approach to Rheumatoid Arthritis. Rheum Dis Clin North Am 2019;45:487-504.

5. Finckh A, Liang MH, van Herckenrode CM, de Pablo P. Long-term impact of early treatment on radiographic progression in rheumatoid arthritis: A meta-analysis. Arthritis Rheum 2006;55:864-72.

6. Martins FM, da Silva JA, Santos MJ, Vieira-Sousa E, Duarte C, Santos H, Costa JA, Pimentel-Santos FM, Cunha I, Cunha Miranda L, Novoa T, Cruz M, Bernardes M, Araujo D, Pereira Silva JA, Silva JC, Branco JC, Gomes JA, Faustino A, Fonseca JE, Canhao H. DAS28, CDAI and SDAI cut-offs do not translate the same information: results from the Rheumatic Diseases Portuguese Register Reuma.pt. Rheumatology (Oxford) 2015;54:286-91.

7. Kim JY, Cho SK, Han M, Choi YY, Bae SC, Sung YK. The role of bone scintigraphy in the diagnosis of rheumatoid arthritis according to the 2010 ACR/EULAR classification criteria. J Korean Med Sci 2014;29:204-9.

8. Colebatch AN, Edwards CJ, Ostergaard M, van der Heijde D, Balint PV, D'Agostino MA, Forslind K, Grassi W, Haavardsholm EA, Haugeberg G, Jurik AG, Landewe RB, Naredo E, O'Connor PJ, Ostendorf B, Potocki K, Schmidt WA, Smolen JS, Sokolovic S, Watt I, Conaghan PG. EULAR recommendations for the use of imaging of the joints in the clinical management of rheumatoid arthritis. Ann Rheum Dis 2013;72:804-14.

9. Mandl P, Naredo E, Wakefield RJ, Conaghan PG, D'Agostino MA. A systematic literature review analysis of ultrasound joint count and scoring systems to assess synovitis in rheumatoid arthritis according to the 
OMERACT filter. J Rheumatol 2011;38:2055-62.

10. Wong LM, Shi L, Xiao F, Griffith JF. Fully automated segmentation of wrist bones on T2-weighted fatsuppressed MR images in early rheumatoid arthritis. Quant Imaging Med Surg 2019;9:579-89.

11. Grant FD, Fahey FH, Packard AB, Davis RT, Alavi A, Treves ST. Skeletal PET with 18F-fluoride: applying new technology to an old tracer. J Nucl Med 2008;49:68-78.

12. Czernin J, Satyamurthy N, Schiepers C. Molecular mechanisms of bone $18 \mathrm{~F}-\mathrm{NaF}$ deposition. J Nucl Med 2010;51:1826-9.

13. Segall G, Delbeke D, Stabin MG, Even-Sapir E, Fair J, Sajdak R, Smith GT. SNM practice guideline for sodium 18F-fluoride PET/CT bone scans 1.0. J Nucl Med 2010;51:1813-20.

14. Suh MS, Park SH, Kim YK, Yun PY, Lee WW. (18)F-NaF PET/CT for the evaluation of temporomandibular joint disorder. Clin Radiol 2018;73:414.e7-414.e13.

15. Strobel K, Fischer DR, Tamborrini G, Kyburz D, Stumpe KD, Hesselmann RG, Johayem A, von Schulthess GK, Michel BA, Ciurea A. 18F-fluoride PET/CT for detection of sacroiliitis in ankylosing spondylitis. Eur J Nucl Med Mol Imaging 2010;37:1760-5.

16. Lee JW, Park YJ, Jeon YS, Kim KH, Lee JE, Hong SH, Lee SM, Jang SJ. Clinical value of dual-phase F-18 sodium fluoride PET/CT for diagnosing bone metastasis in cancer patients with solitary bone lesion. Quant Imaging Med Surg 2020;10:2098-111.

17. Sheikhbahaei S, Jones KM, Werner RA, Salas-Fragomeni RA, Marcus CV, Higuchi T, Rowe SP, Solnes LB, Javadi MS. (18)F-NaF-PET/CT for the detection of bone metastasis in prostate cancer: a meta-analysis of diagnostic accuracy studies. Ann Nucl Med 2019;33:351-61.

18. Lee JW, Yu SN, Yoo ID, Jeon MH, Hong CH, Shim JJ, Chang SH, Lee SM. Clinical application of dual-phase F-18 sodium-fluoride bone PET/CT for diagnosing surgical site infection following orthopedic surgery. Medicine (Baltimore) 2019;98:e14770.

19. Tan AL, Tanner SF, Waller ML, Hensor EM, Burns A, Jeavons AP, Bury RF, Emery P, McGonagle D. Highresolution $[18 \mathrm{~F}]$ fluoride positron emission tomography of the distal interphalangeal joint in psoriatic arthritis-a bone-enthesis-nail complex. Rheumatology (Oxford) 2013;52:898-904.

20. Raynal M, Bouderraoui F, Ouichka R, Melchior J, Morel O, Blum A, Chary-Valckenaere I, Ngueyon Sime W, Roch V, Maksymowych W, Lambert RG, Olivier P, Loeuille D. Performance of (18)F-sodium fluoride positron emission tomography with computed tomography to assess inflammatory and structural sacroiliitis on magnetic resonance imaging and computed tomography, respectively, in axial spondyloarthritis. Arthritis Res Ther 2019;21:119.

21. Watanabe T, Takase-Minegishi K, Ihata A, Kunishita Y, Kishimoto D, Kamiyama R, Hama M, Yoshimi R, Kirino Y, Asami Y, Suda A, Ohno S, Tateishi U, Ueda A, Takeno M, Ishigatsubo Y. (18)F-FDG and (18)F-NaF PET/CT demonstrate coupling of inflammation and accelerated bone turnover in rheumatoid arthritis. Mod Rheumatol 2016;26:180-7.

22. Jonnakuti VS, Raynor WY, Taratuta E, Werner TJ, Alavi A, Baker JF. A novel method to assess subchondral bone formation using $[18 \mathrm{~F}] \mathrm{NaF}-\mathrm{PET}$ in the evaluation of knee degeneration. Nucl Med Commun 2018;39:451-6.

23. Lee JW, Na JO, Kang DY, Lee SY, Lee SM. Prognostic Significance of FDG Uptake of Bone Marrow on PET/ CT in Patients With Non-Small-Cell Lung Cancer After Curative Surgical Resection. Clin Lung Cancer 2017;18:198-206.

24. Scheel AK, Hermann KG, Kahler E, Pasewaldt D, Fritz J, Hamm B, Brunner E, Müller GA, Burmester GR, Backhaus M. A novel ultrasonographic synovitis scoring system suitable for analyzing finger joint inflammation in rheumatoid arthritis. Arthritis Rheum 2005;52:733-43.

25. Szkudlarek M, Court-Payen M, Jacobsen S, Klarlund M, Thomsen HS, Østergaard M. Interobserver agreement in ultrasonography of the finger and toe joints in rheumatoid arthritis. Arthritis Rheum 2003;48:955-62.

26. Irmler IM, Gebhardt P, Hoffmann B, Opfermann T, Figge MT, Saluz HP, Kamradt T. 18 F-Fluoride positron emission tomography/computed tomography for noninvasive in vivo quantification of pathophysiological bone metabolism in experimental murine arthritis. Arthritis Res Ther 2014;16:R155.

27. do Prado AD, Staub HL, Bisi MC, da Silveira IG, Mendonca JA, Polido-Pereira J, Fonseca JE. Ultrasound and its clinical use in rheumatoid arthritis: where do we stand? Adv Rheumatol 2018;58:19.

28. Möller B, Aletaha D, Andor M, Atkinson A, AubryRozier B, Brulhart L, Dan D, Finckh A, Grobéty V, Mandl P, Micheroli R, Nissen MJ, Nydegger AM, Scherer A, Tamborrini G, Ziswiler HR, Zufferey P; SONAR group for musculoskeletal ultrasound in the Swiss Clinical Quality Management (SCQM) Foundation. Synovitis in rheumatoid arthritis detected by grey scale ultrasound predicts the development of erosions over the next three 
years. Rheumatology (Oxford) 2020;59:1556-65.

29. van Riel PL, Renskers L. The Disease Activity Score (DAS) and the Disease Activity Score using 28 joint counts (DAS28) in the management of rheumatoid arthritis. Clin Exp Rheumatol 2016;34:S40-4.

30. Zhang $\mathrm{H}, \mathrm{Xu} \mathrm{H}$, Chen $\mathrm{S}, \mathrm{Mao} \mathrm{X}$. The application value of MRI in the diagnosis of subclinical inflammation in patients with rheumatoid arthritis in remission. J Orthop Surg Res 2018;13:164.

31. Orange DE, Agius P, DiCarlo EF, Mirza SZ, Pannellini T, Szymonifka J, Jiang CS, Figgie MP, Frank MO, Robinson WH, Donlin LT, Rozo C, Gravallese EM, Bykerk VP, Goodman SM. Histologic and Transcriptional Evidence of Subclinical Synovial Inflammation in Patients With Rheumatoid Arthritis in Clinical Remission. Arthritis Rheumatol 2019;71:1034-41.

32. Lillegraven S, Haavardsholm EA. Subclinical Treatment Targets in Rheumatology: Lessons from Randomized Clinical Trials in Rheumatoid Arthritis. Rheum Dis Clin North Am 2019;45:593-604.

33. Foltz V, Gandjbakhch F, Etchepare F, Rosenberg C, Tanguy ML, Rozenberg S, Bourgeois P, Fautrel B. Power Doppler ultrasound, but not low-field magnetic resonance imaging, predicts relapse and radiographic disease progression in rheumatoid arthritis patients with low levels of disease activity. Arthritis Rheum 2012;64:67-76.

34. Alasaarela E, Suramo I, Tervonen O, Lahde S, Takalo R, Hakala M. Evaluation of humeral head erosions in rheumatoid arthritis: a comparison of ultrasonography, magnetic resonance imaging, computed tomography and plain radiography. Br J Rheumatol 1998;37:1152-6.

35. Baker JF, Conaghan PG, Gandjbakhch F. Update on magnetic resonance imaging and ultrasound in rheumatoid arthritis. Clin Exp Rheumatol 2018;36 Suppl 114:16-23.

36. Jindal S, Kaushik R, Raghuvanshi S, Kaushik RM, Kakkar R. Gray Scale and Power Doppler Ultrasonographic Findings in the Assessment of Disease Activity and Their Correlation with Disease Activity Parameters in Rheumatoid Arthritis. Curr Rheumatol Rev 2018;14:153-62.

37. Ostergaard M, Boesen M. Imaging in rheumatoid arthritis: the role of magnetic resonance imaging and computed tomography. Radiol Med 2019;124:1128-41.

38. Kim JY, Choi YY, Kim CW, Sung YK, Yoo DH. Bone Scintigraphy in the Diagnosis of Rheumatoid Arthritis: Is There Additional Value of Bone Scintigraphy with Blood Pool Phase over Conventional Bone Scintigraphy? J Korean Med Sci 2016;31:502-9.

39. Choi HJ, Lee SJ, Kim JY, Sung YK, Choi YY. The
Correlation Between Tenosynovitis Pattern on Two-Phase Bone Scintigraphy and Clinical Manifestation in Patients with Suspected Rheumatoid Arthritis. Nucl Med Mol Imaging 2019;53:278-86.

40. de Bois MH, Arndt JW, van der Velde EA, Pauwels EK, Breedveld FC. Joint scintigraphy for quantification of synovitis with $99 \mathrm{~m}$ Tc-labelled human immunoglobulin $\mathrm{G}$ compared to late phase scintigraphy with $99 \mathrm{mTc}$-labelled diphosphonate. Br J Rheumatol 1994;33:67-73.

41. Ohrndorf S, Fischer IU, Kellner H, Strunk J, Hartung W, Reiche B, Burmester GR, Walther M, Schmidt WA, Backhaus M. Reliability of the novel 7-joint ultrasound score: results from an inter- and intraobserver study performed by rheumatologists. Arthritis Care Res (Hoboken) 2012;64:1238-43.

42. Saboury B, Parsons MA, Moghbel M, Rubello D, Brothers A, Torigian DA, Werner TJ, Houshmand S, Basu S, Lam MG, Alavi A. Quantification of aging effects upon global knee inflammation by 18 F-FDG-PET. Nucl Med Commun 2016;37:254-8.

43. Bhattarai A, Nakajima T, Sapkota S, Arisaka Y, Tokue A, Yonemoto Y, Tsushima Y. Diagnostic value of $18 \mathrm{~F}$-fluorodeoxyglucose uptake parameters to differentiate rheumatoid arthritis from other types of arthritis. Medicine (Baltimore) 2017;96:e7130.

44. Khaw TH, Raynor WY, Borja AJ, Al-Zaghal A, Jonnakuti VS, Cheng N, Houshmand S, Werner TJ, Alavi A.

Assessing the effects of body weight on subchondral bone formation with quantitative (18)F-sodium fluoride PET. Ann Nucl Med 2020;34:559-64.

45. Lee SJ, Jeong JH, Lee CH, Ahn BC, Eun JS, Kim NR, Kang JW, Nam EJ, Kang YM. Development and Validation of an (18) F-Fluorodeoxyglucose-Positron Emission Tomography With Computed TomographyBased Tool for the Evaluation of Joint Counts and Disease Activity in Patients With Rheumatoid Arthritis. Arthritis Rheumatol 2019;71:1232-40.

46. Raynor WY, Jonnakuti VS, Zirakchian Zadeh M, Werner TJ, Cheng G, Zhuang H, Høilund-Carlsen PF, Alavi A, Baker JF. Comparison of methods of quantifying global synovial metabolic activity with FDG-PET/CT in rheumatoid arthritis. Int J Rheum Dis 2019;22:2191-8.

47. Beckers C, Jeukens X, Ribbens C, André B, Marcelis S, Leclercq P, Kaiser MJ, Foidart J, Hustinx R, Malaise MG. (18)F-FDG PET imaging of rheumatoid knee synovitis correlates with dynamic magnetic resonance and sonographic assessments as well as with the serum level of metalloproteinase-3. Eur J Nucl Med Mol Imaging 
2006;33:275-80.

48. Usmani S, Rasheed R, Al Kandari F. Textitis as Seen on (18) F-NaF Imaging Using an Ultra-High-Resolution Positron
Emission Mammography Scanner. J Nucl Med Technol 2020;48:181-3.

Cite this article as: Park HJ, Chang SH, Lee JW, Lee SM. Clinical utility of F-18 sodium fluoride PET/CT for estimating disease activity in patients with rheumatoid arthritis. Quant Imaging Med Surg 2021;11(4):1156-1169. doi: 10.21037/qims20-788 


\section{Supplementary}
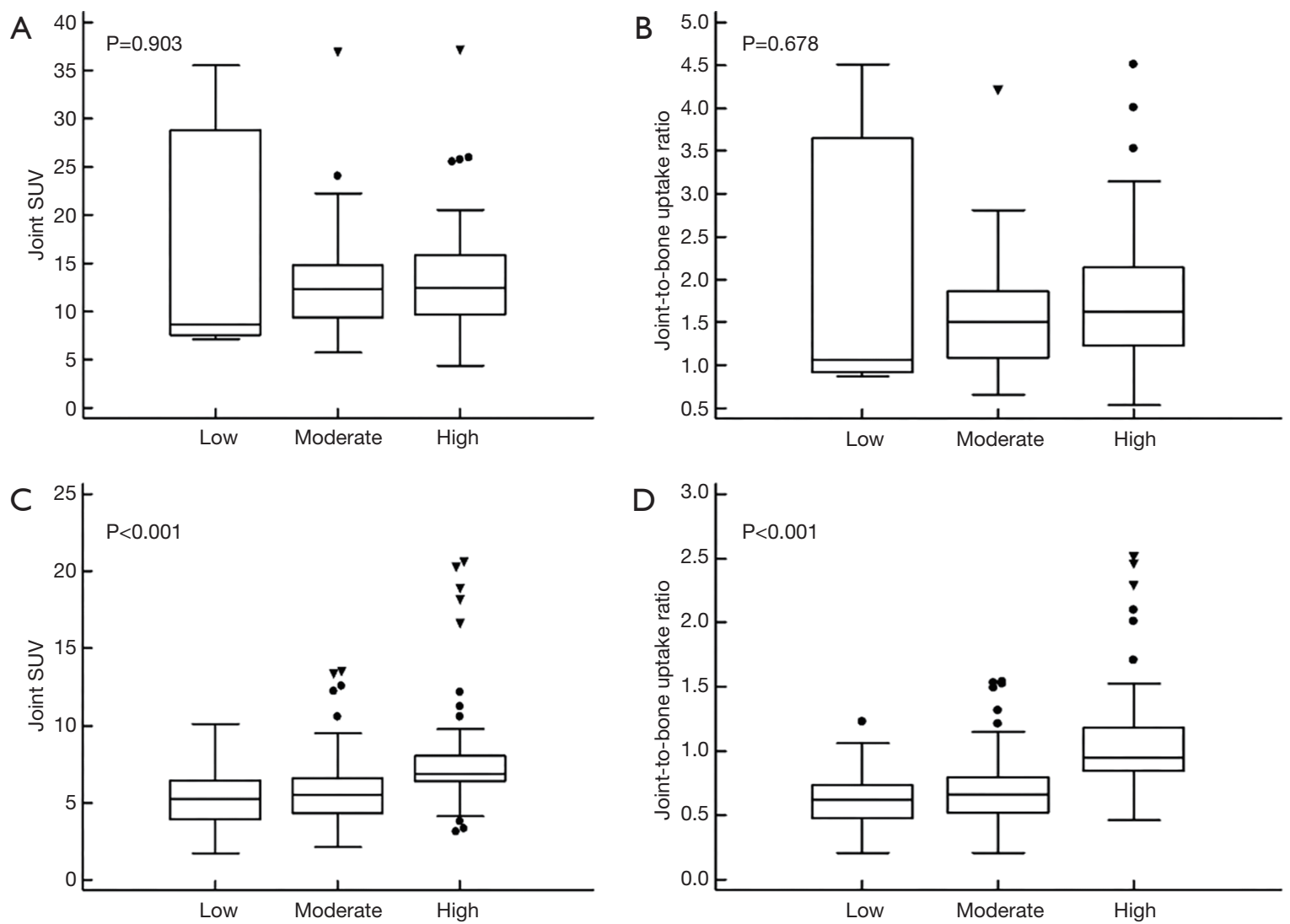

Figure S1 Distribution of the joint SUV (A) and joint-to-bone uptake ratio (B) of the joints with synovitis (tender and/or swollen joints) and distribution of the joint SUV (C) and joint-to-bone uptake ratio (D) of the joints without synovitis according to the disease activity of rheumatoid arthritis. 